\title{
Closing the Gap Between Designers and Developers in a Low Code Ecosystem
}

\author{
Mariana Bexiga \\ NOVA University of Lisbon \\ Portugal \\ md.bexiga@campus.fct.unl.pt
}

\author{
Stoyan Garbatov \\ OutSystems \\ Portugal \\ stoyan.garbatov@outsystems.com
}

\author{
João Costa Seco \\ NOVA University of Lisbon, NOVA \\ LINCS \\ Portugal \\ joao.seco@fct.unl.pt
}

\begin{abstract}
Nowadays, going digital is a must for a company to thrive and remain competitive. The digital transformation allows companies to react timely and adequately to the constantly evolving markets. This transformation is not without challenges. Among these is the growing demand for skilled software developers. Low-code platforms have risen to mitigate this pressure point by allowing people with non-programming backgrounds to craft digital systems capable of solving business relevant problems.

Professional development teams are composed of many different profiles - product owners, analysts, UX and UI designers, front-end and back-end developers, among others. Market competition puts unprecedented demands on the collaboration of these professionals. Current methodologies provide tools and approaches for many of these types of collaboration. However, the reality of established industry practices for UX and UI designers collaborating with frontend developers, still leaves a lot to improve in terms of effectiveness and efficiency.

This work developed an innovative approach using model transformation and meta-modelling techniques that drastically improves the efficiency of transforming UX/UI design artefacts into low-code web-technology. The approach has been applied to a recognized and established enterprise-grade low-code platform and evaluated in practice by a team of professional designers and front-end developers. Preliminary practical results show savings between 20 and $75 \%$ according to the project complexity in the effort invested by development teams in the above mentioned process.
\end{abstract}

\section{CCS CONCEPTS}

- Software and its engineering $\rightarrow$ Application specific development environments.

\section{KEYWORDS}

Low-Code Platform, Design To Code, OutSystems, Front-end Development

Permission to make digital or hard copies of all or part of this work for personal or classroom use is granted without fee provided that copies are not made or distributed for profit or commercial advantage and that copies bear this notice and the full citation on the first page. Copyrights for components of this work owned by others than ACM must be honored. Abstracting with credit is permitted. To copy otherwise, or republish, to post on servers or to redistribute to lists, requires prior specific permission and/or a fee. Request permissions from permissions@acm.org.

MODELS '20 Companion, October 18-23, 2020, Virtual Event, Canada

(C) 2020 Association for Computing Machinery.

ACM ISBN 978-1-4503-8135-2/20/10 . \$ $\$ 15.00$

https://doi.org/10.1145/3417990.3420195
ACM Reference Format:

Mariana Bexiga, Stoyan Garbatov, and João Costa Seco. 2020. Closing the Gap Between Designers and Developers in a Low Code Ecosystem. In ACM/IEEE 23rd International Conference on Model Driven Engineering Languages and Systems (MODELS '20 Companion), October 18-23, 2020, Virtual Event, Canada. ACM, New York, NY, USA, 10 pages. https://doi.org/10.1145/ 3417990.3420195

\section{INTRODUCTION}

The number of people using digital systems has grown significantly in the last decades and so have their high expectations in terms of usability, reliability and performance. This has led the development of highly polished user interfaces to become an ever growing priority for companies. Good user experience is a crucial factor for setting a product apart from the competition. Understanding users and paying attention to their interaction with systems is even more determinant in the end-user market.

Software development requires the collaboration of a wide range of professionals. Collaborative work processes help designing systems that meet user needs in an effective and efficient way. However, groups involved in software development use distinct methodologies, tools and constraints, whose differences hinder the project's productivity and efficiency. The transition between the design of user interfaces and user experience phases and its implementation by the front-end developers is one of those cases.

The implementation of design specifications is based on highfidelity representations created by specialized designers using dedicated tools. The process is performed manually and even for expert developers the conversion from design artifacts to web technology applications is not a trivial task. The diversity of components in UI designs makes the conversion a complex process. Designers and developers iterate to produce different UI versions for multiple contexts using their own tools until they are accepted by clients. This is a cumbersome, time-consuming and error-prone process and the final result is frequently different from what was designed.

This work is inserted in the OutSystems ecosystem, a low-code development platform where the processing of the UI design artifacts is done by front-end developers, who manually convert UI elements and corresponding customization to their OutSystems representation. This is a manual process and sometimes customization is not translated properly, making the end result different from the initial design. Furthermore, several stages of the translation process are common to all projects and perceived as monotonous and not challenging by front-end specialists. Nonetheless, these challenges are not specific to OutSystems and other technology ecosystems experience similar challenges. 
Having a mechanism able to translate design specifications and applications look-and-feel from design tools to front-end code significantly improves the workflow productivity and reduces errors in the transition process. The design to code translation problem is not only relevant in the low-code platforms domain but also to other web technologies, where there are also limited opportunities to automatically convert artifacts.

Our approach is based on an abstract intermediate representation and meta-model to process and create models. It relies on model transformations to successfully convert UI design models made with a visual domain language [5] to an abstract intermediate representation. Later, the intermediate artifact is transformed to the corresponding OutSystems [20] customized applications. Our approach is materialized through a prototype tool that receives a template application containing all base elements as input and generates customized OutSystems applications. The use of the tool represents a significant reduction in the duration of the conversion and customization process carried out by professional teams, which can reach $30 \%$ and a reduction from 5 to 3 days in the typical development project planning.

Even though we are covering the Sketch to OutSystems conversion, due to the generic and technology-independent nature of the solution architecture, this approach can be applied to other design and web technologies.

The remaining paper is organized as follows. Section 2 reviews related work and Section 3 describes in detail the source and target models of the solution. Section 4 outlines our approach to convert design artifacts made with design tools to its web technology representation using an intermediate representation. The section also highlights the challenges of modeling and transforming both models as well as the evaluation of the tool. Section 5 covers the concluding remarks as well as relevant future work.

\section{RELATED WORK}

The automatic translation from design artifacts to front-end representations field is maturing and different tools and approaches have emerged recently.

We analyzed multiple approaches that aim to solve the UI to web technology conversion problem. Such techniques often rely on heuristics, computer vision and optical character recognition $[6,8,19]$, object detection algorithms $[15,25]$ and neural networks that cover the process from end to end and are trained to understand the input and generate the corresponding code $[3,14]$. These three groups of tools take images or hand-drawn sketches as inputs. However, real-world design processes are performed using design tools and the input of these approaches are high-fidelity representations of the system. That being said, the approaches described above are somehow disconnected from the established industry practices. Our approach is conceptually different from the above works since it relies on structured inputs for UI design artifacts.

New languages have emerged to model user interfaces, namely User Interface Markup Languages. These languages are target independent languages for modeling UI and UX that help mitigating the conversion problem, improving the design to front-end transition. Several examples are XML-based languages such as XIML [22], UIML [1], and USIXML [16]. These languages ease the automatic generation of code and do not require extra effort to adapt code to multiple platforms [23]. Multi-path UI development has been defined as an engineering method and tool that allows a designer to start a UI development by several entry points in the development cycle, and from these entry points get substantial support to build a high quality UI. This requires the full adoption of code specification languages in the workflow of designers and in practice, designers do not adopt such approaches.

IFML [4] is a standard modeling language to create graphical representations of interfaces and behavior for desktop, mobile and client-server applications. The language helps to model different front-end aspects such as navigation paths, user events and interaction and supports connection with the business logic, the data model and the graphical presentation layer. However, IFML is not concerned with the appearance of applications but with their structure and behaviour. The code generated by IFML only concerns the structural organization of elements, interaction with the system and events.

Several commercial tools in the market support design and development processes separately, however, they do not provide mechanisms for smoothly interfacing the work from designers to developers. In the last few years, new tools focused on converting design artifacts to code emerged whose main goal is to minimize the gap between design and front-end development. However, the existing conversion tools [12,17, 21, 24, 26,29] do not have mechanisms to generate low-code artifacts and consequently do not support the growing trends of the low-code platform market.

On the other hand, most low-code platforms [2, 9, 11, 27, 28, 30] do not have mechanisms to address the inefficiencies in the design to code conversion process and do not allow users to smoothly transition from design tools to low-code platforms. Some platforms do not have design assets for design tools making the conversion process even harder since designed components have no connection to the low-code platform UI widgets. Design tools such as Figma [7] and InVision [10] do have code generation mechanisms and plenty of low-code platforms encourage their usage as a helper in the transition process. In the OutSystems system ecosystem, the code generated by these tools is not used by professional teams since they do not export to low-code web technology.

\section{OUR APPROACH}

As a way to promote consistency, OutSystems created Live Style Guides (LSG) libraries where all the documentation and guidelines regarding the appearance behavior of projects is organized. LSG applications are modules that contain the most commonly used UI components of an OutSystems application. By customizing the state of such elements a team ensures a uniform development of UI during a project and across several projects for a given line-of-business or even a company. Beside basic styling rules, the LSG application includes ready-to-use UI Patterns and Widgets already customized with the client branding according to the OutSystems standards. These repositories are used by both designers and developers. Every time application pages need to be created or changed, there is no need to create or customize widgets from scratch. Not only the look of components is described but also their behavior. Live Style Guides abstract CSS and JavaScript complexity from developers. 
Live Style Guides contain more than 70 reusable UI components and for the majority of applications no new patterns need to be created. However, new custom-made patterns not defined in the framework can be added.

Within the OutSystems community, LSG are widely promoted and used by both OutSystems professional teams and partner companies $[13,18]$ that work with the OutSystems technology. Their usage promotes reusability and consistency. Maintenance costs of projects are reduced and development velocity increased when building pages and patterns.

To better understand the work dynamics in the OutSystems ecosystem, we interviewed 25 professionals involved in the delivery of OutSystems enterprise-grade applications. The interviewed group included not only designers and developers from OutSystems but also other roles such as Project Managers, Tech Leads, Team Leads, People Allocation Managers, among others involved in delivering a project successfully. The main objective was to understand what are the main steps of a project, the tools and approaches used as well as the most frequent challenges and their implications.

The OutSystems Customer Success department is responsible for carrying out projects for OutSystems customers. It is frequent for customers who are new to OutSystems technology or do not have dedicated development teams to employ the services provided by this department. The usual flow in a project carried out by the OutSystems Customer Success department is divided into 4 main phases: Initiation, UI Design, Live Style Guide (LSG) Creation and Development.

The transition between UI Design and the LSG Creation is the focus of our work. In the UI Design phase, UI designers convert low-fidelity mockups of the system produced by UX designers into more detailed representations and the process usually takes 3 days. UI designers create high-fidelity mockups using design tools such as Sketch [5], Figma [7] or InVision[10].

Based on the high-fidelity mockups, front-end developers are responsible for developing OutSystems Live Style Guide applications and sample pages based on the high-fidelity mockups previously done by designers. Nevertheless there are no tools or mechanisms to help and ease the conversion process in the OutSystems context. Translation is performed manually by a front-end developer and has a standard duration of 5 days. The artifacts produced are two OutSystems applications corresponding to the Theme and the Live Style Guide. The Theme Application has project styles and custom patterns and goes to production. The modules included are the Theme module with the CSS, Resources module with fonts and images and Patterns module with specific patterns not built on the OutSystems low-code UI framework for web and mobile applications. The Live Style Guide application includes documentation of the project styles and custom patterns and does not go to production. The application includes the Template module that can be used when a new application is created and the Live Style Guide module that includes a live demo of the OutSystems UI and custom Patterns.

\subsection{Base Models}

The source and target models of our approach were selected based on the development process used at OutSystems explained in section 3. As the most used design tool in the ecosystem is Sketch, the representation of Sketch visual design language was chosen as the source model. Since the project is inserted in the OutSystems context, the target model of our solution is the OutSystems app model.

To validate and illustrate our proposal on how to transform design artifacts into web technology artifacts we are using Sketch and OutSystems as examples. However, the solution architecture proposed is designed to be generic and not to be limited to on a specific design or web technology. This way, in the future it will be easy to scale and generalize for other technologies.

Other design tools, such as Figma [7] have similar meta-models in comparison with Sketch [5]. Consequently, their processing and manipulating would require a similar approach.

\subsection{Sketch Visual Domain-Specific Language}

Sketch [5] is a vector graphic editor used by designers to produce high-fidelity representations of user interfaces. The Sketch language is a visual Domain-Specific Language where every element is represented by a Layer. Different types of layers are characterized by a different set of elements and attributes. The most relevant attributes and classes for our tool from a Sketch file are shown in Figure 1.

Some of the most relevant types of layers that compose the Sketch concrete syntax and that are frequently used in the process we are studying are:

- Group - collection of 1 or more layers. A group is a type of layer used for organizational purposes.

- Page - instance of a group layer representing a canvas in the document.

- Artboard - instance of a group layer representing a collection of layers.

- Image - layer whose content is an image file

- Shape - most common layer type. Shape layers are used for introducing pre-made or new shapes on the document.

- Text - layer whose content is a text element

- Symbol Master - instance of an Artboard (and consequently of a Group layer). Layers frequently used across the document can be promoted to symbols. Every time changes are made in the Symbol master, modifications are propagated to all its instances.

- Symbol Instance - instance of a Symbol Master layer. Symbol instance layers have a reference for their master and keep its structure. However, attributes of the inner layers of a Symbol Instance may be changed such as colors, shapes or text styles. A Symbol Instance layer stores the new attribute values as Override Values, elements of the Symbol whose value is changed in relation to the original Symbol Master.

Layers are characterized by a group of elements:

- Frame - contains the dimensions of the element as well as its coordinates in the parent layer. 


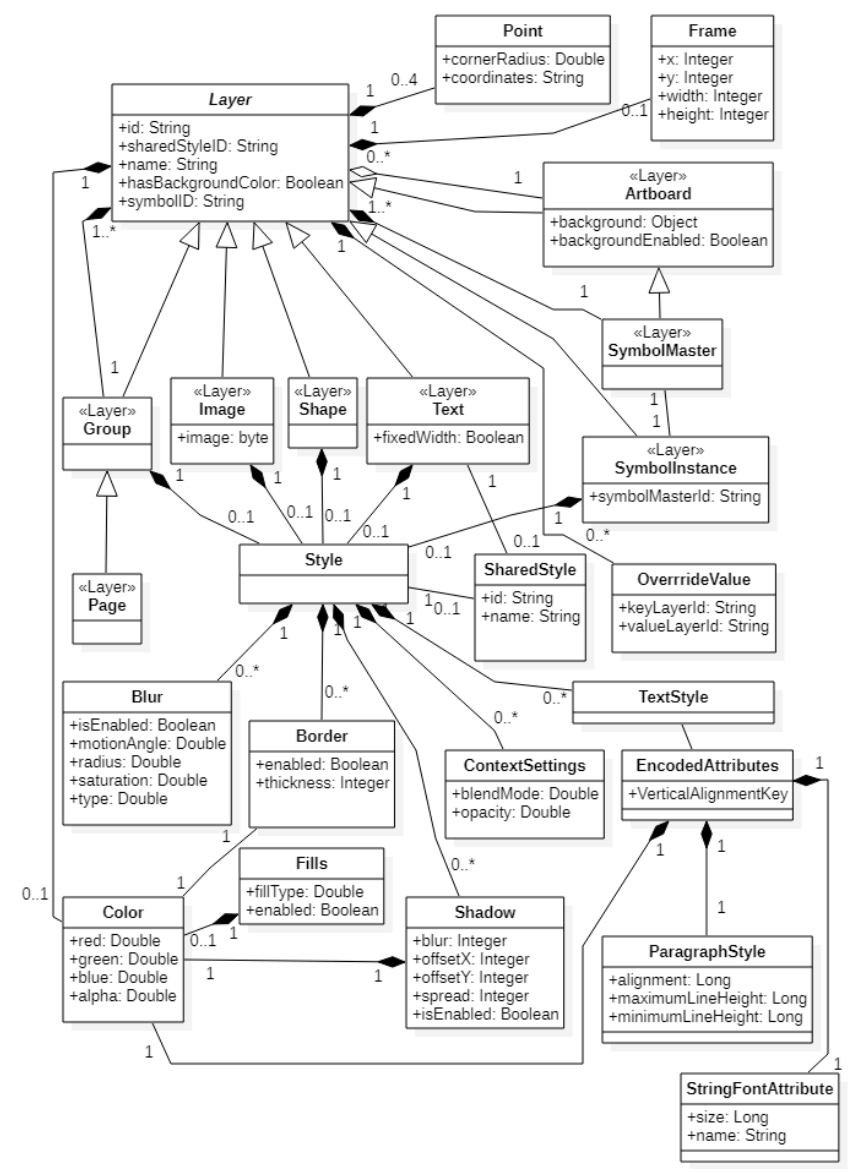

Figure 1: Sketch Page Domain Meta-model

- Points - describes the radius of the four corners of the layer. A layer can have 4 points representing the 4 corners or no points when the layer is a rectangle.

- Color - represents the layer background color. If the background color is transparent, the layer has no color.

- Override Value - a layer can have elements of the Symbol whose value is changed in relation to the original Symbol Master. The layer Override Value is a list of pairs where the key represents the original inner layer of the symbol master and the value its new value.

Group, Image, Shape, Text and Symbol Instance Layers have Style. Styles are composed by Blur, Shadow, Border, Text Styles and Shared Styles.

\subsection{The Live Style Guide Sketch Model}

The Sketch visual language is powerful and its notation allows the creation of a wide variety of compositions. However, there is no one-to-one mapping between design concepts, namely those used in Sketch and the web technology UI elements.

For the reasons mentioned above, we restricted Sketch's modeling space to a Sketch file that works as a template. The use of this file as a sharing point facilitates the identification of the elements.

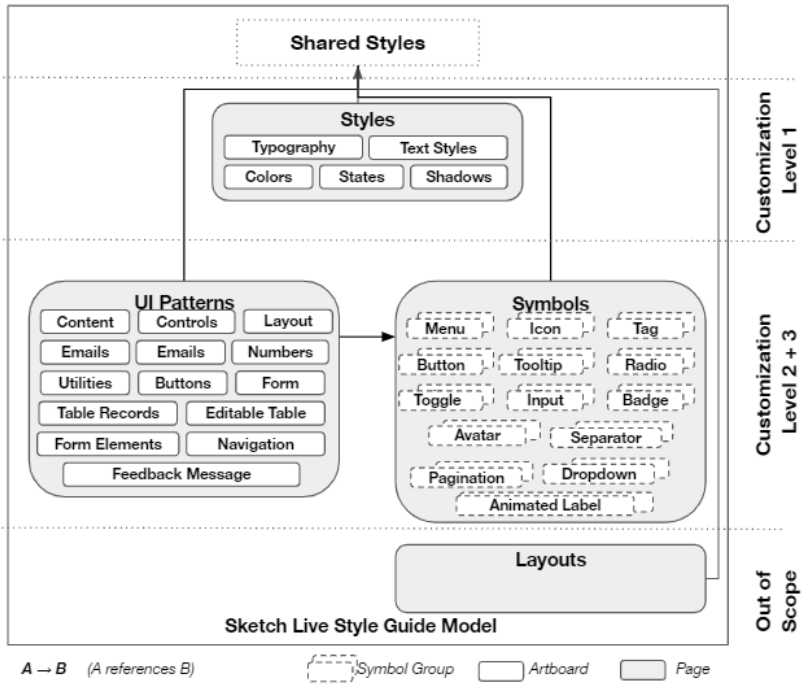

Figure 2: The Sketch LSG Model

Mapping a group of layers to a widget would not be possible without a template and using a model-based methodology given the variety of Sketch trees allowed to represent the component.

The file used as our Sketch template contains representations of every component present in the OutSystems UI framework crafted on Sketch. The file was developed during an extended period of time by the OutSystems UI team and corresponds to a continuous optimization of the current framework. Whenever the framework is updated, the file is also updated. In enterprise projects that follow the established practices, the Sketch file is used as a base for UI designers to customize components according to the client branding. Our source model is the current version of the Live Style Guide Sketch file.

The Live Style Guide Sketch file structure is illustrated in Figure 2. The document is divided into four pages:

3.3.1 Styles. The Styles Page contains base design information divided into 5 artboards. Each artboard has information about an element, namely, typography, colors, shadow styles, states and text styles. The processing and mapping of the Styles Page covers the first level of application customization explained in Section 3.5.

3.3.2 Symbols. The Symbols Page contains all the Symbol Master Layers representing logos, headers, buttons among others. The symbols in this page are customized with the styles defined in the Styles Pages. Instances of the symbols defined in the Symbols page are used in the Layouts and UI Patterns/Widgets pages. Every time the master symbol is edited, changes are made to every instance.

3.3.3 UI Patterns and Widgets. The UI Patterns and Widgets page contains OutSystems UI Patterns and Widgets divided into 13 artboards. Every component is represented by multiple instances representing all the widget states such as visited, hover or filled.

However, depending on the LSG Sketch template version, the organization of the document varies slightly. so that the tool can process different versions, the processing of the UI Patterns and 


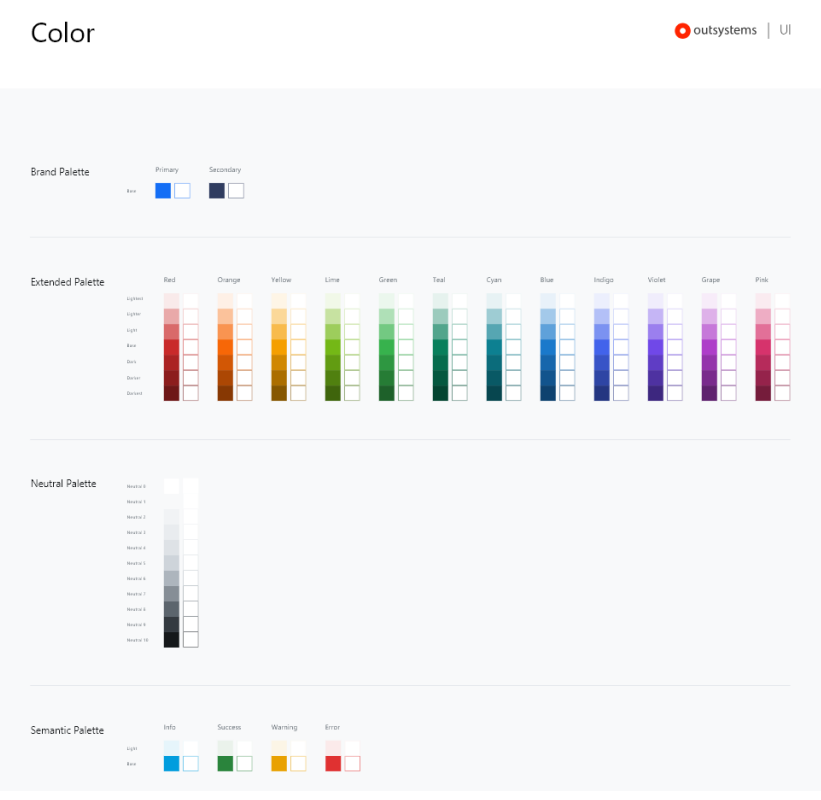

Figure 3: Color Artboard of the Sketch LSG

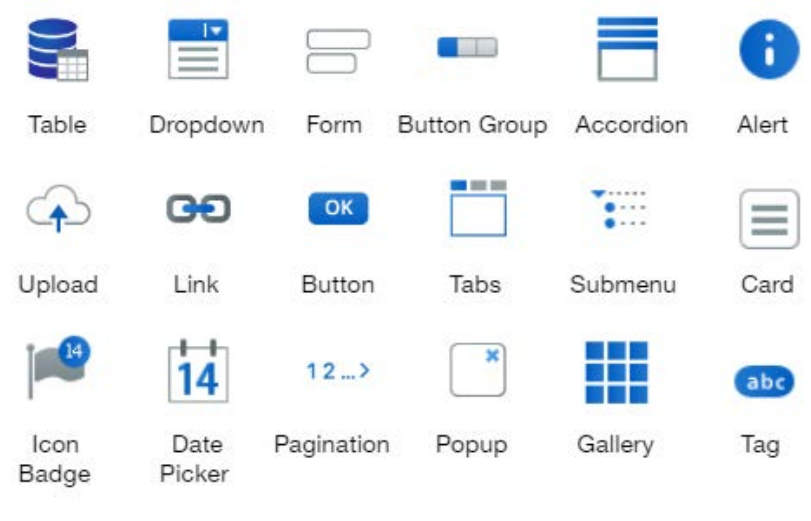

Figure 4: Concrete Syntax of the OutSystems UI DSL

Widgets pages is done by identifying the groups present in each artboard and not the default organization of each page. Therefore, the tool can handle structurally different formats as long as its semantics are equivalent.

3.3.4 Layouts. The Layout page includes pre-build screen components arrangements such as Login, Top Menu and Side Menu.

Besides these pages, the model contains a set of style rules that can be applied and shared across the document layers named Shared Styles. The usage of these style elements makes the process of customizing layers easier and eliminates the need to re-do styles. Every time a layer uses a Shared Style item, they have a reference to it. The Shared Styles can be found in one of the JSON files.

\subsection{The OutSystems Model}

The OutSystems language is a strongly typed language composed by a group of visual domain-specific languages that model all the application layers: processes, interfaces, logic and data.

In OutSystems, applications' user interfaces are named Web Screens and are organized hierarchically. A group of constructs of the Interfaces DSL concrete syntax is illustrated in Figure 4. Interface modeling is done by drag-and-drop of widgets and other elements to the OutSystems IDE, Service Studio.

A portion of the Interfaces DSL constructs are screens building blocks. The elements are named Widgets and some examples are lists or dropdowns. The selection of widgets represents the most common user interface elements. Every widget is described by a set of properties that model the widget according to the application. The properties include source data, behavior and appearance among others.

Besides widgets, the Interface DSL includes components that are pragmatic or have control for defining applications. Some examples are Conditionals and Expressions.

\subsection{Interfaces Customization using OutSystems}

The widgets described above compose the OutSystems UI framework. The framework defines UI Patterns and Widgets structure and look-and-feel. The predefined appearance of a new application is inherited from OutSystems UI. However, changing the application or widget appearance can be done by writing additional Cascading Style Sheets (CSS) or JavaScript.

In the customization process described in section 3, we identified a process of transforming a template LSG to a fully customized app that can be used in the project. Applications customization can be divided into 4 levels:

- Level 1 - The simplest level of customization comprises the customization of CSS styles. Style customization includes overriding variables declared in the OutSystems UI framework values. The element group includes colors, typography, text styles, states and shadows.

- Level 2 - Customization that includes overriding the styling rules defined by the OutSystems UI framework. In this level, no new properties are added to elements.

- Level 3 - Customization that goes beyond the attributes defined in the OutSystems Design System. New CSS rules are added, and, in existing CSS rules, new properties can be added.

- Level 4 - Customization that changes the default UI pattern structure and is not contemplated in the Level 3. The creation of custom Widgets and UI Patterns is included in this level.

\subsection{Tool Functioning Overview}

Our main goal is to automate the translation of high-fidelity design artifacts to OutSystems applications conversion, described in Section 3, thus reducing the time invested in this step and the amount of inconsistencies and errors introduced. The way the solution architecture was designed allows for scalability for other technologies in the future since it does not rely on a specific design or web technology. 


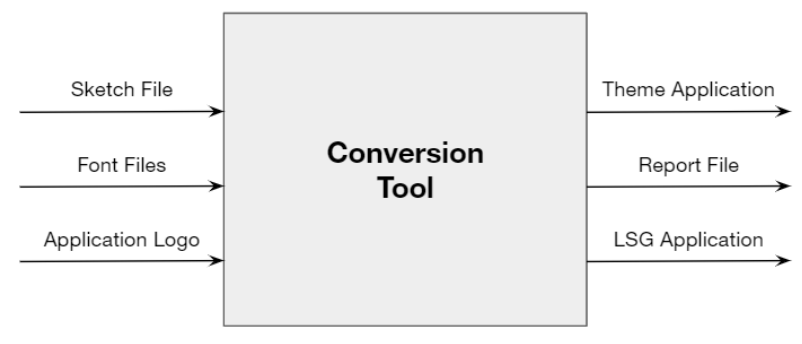

Figure 5: Black-box diagram

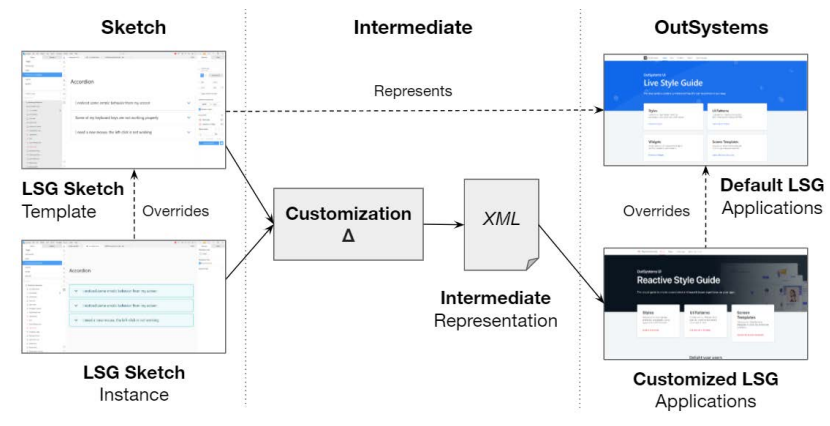

Figure 6: Tool High-Level Concepts

We aim to transform a design tool file instance to instances of the OutSystems model. Taking into account that both models are based on the OutSystems UI design system, we are modeling the customization made upon it and using it to generate customized OutSystems applications.

The system inputs and outputs are illustrated in Figure 5. As inputs, our tool receives an OutSystems LSG Sketch file instance, .ttf or .woff files related to the applications fonts and finally an image representing the application logo. The outputs of our tool are two customized OutSystems applications that represent the LSG and a report file. The report file specifies which components were properly customized and which components require manual intervention.

The high level concepts manipulated by our tool are shown in Figure 6. The architecture is divided into three main groups, Sketch, Intermediate and OutSystems.

As concluded from the initial interviews, the flow of information in the OutSystems ecosystem occurs in one direction, from the design to web representation. For this reason, our tool performs a one-shot unidirectional model transformation from left to right in the diagram of Figure 6.

A Sketch file is given as input. The file is an instance of the LSG Sketch file which means that it overrides the default LSG Sketch file with the client branding. The difference between the two files (Customization $\Delta$ ) is identified and the Customization $\Delta$ calculated. The Customization $\Delta$ is later used to create the Intermediate Representation. Later, the representation is transformed and used to customize an instance of the OutSystems Live Style Guide.

\footnotetext{
${ }^{0}$ Difference between the Sketch instance and the LSG template
}

Sketch documents are compressed archives containing JSON encoded data. For each Sketch page is generated a JSON file. Before being processed by our tool, the Sketch file is unzipped and JSON files extracted. Then, the mapping is carried out between the JSON files obtained and the page to which they correspond. The tool functioning and the steps described previously are explained with detail in the following subsections.

The transformation process is exemplified in Figure 7. The Sketch representation of a Button Group is transformed into its intermediate representation that is finally transformed into the resulting OutSystems LSG Button Group widget. Figure 8 presents the default styling inherited from the OutSystems UI framework.

\subsection{Customization Processing}

The actual processing of each file follows the identification of those different pages of the document. The Sketch processing is divided into two steps, Styles Processing and UI Patterns/Widgets Processing.

3.7.1 Styles Processing. The Styles Processing stage corresponds to the first level of customization. Mapping styles concepts is straightforward since elements needed to process are all available and only 1 layer processing is required. No Symbol layers are used and consequently no extra processing and layer recognition tasks are needed. The design concepts match with the web technology concepts.

Styles concepts include Colors, States, Typography, Text Styles and Shadows. Elements position and label in the page hierarchy is enough to process nodes since models do not suffer changes to its structure.

Widgets and UI Patterns are represented in the corresponding Sketch page and distributed across multiple artboards. The structure illustrated in The Sketch LSG Model corresponds to LSG Sketch template version 1.0.0. However, different versions are organized differently. In order to accommodate those variations, processing is done through the groups of artboards and not by the organization of artboards.

Unlike Style elements, UI Patterns and Widgets are represented in our source model as trees of layers. Processing such elements implies identifying, processing and mapping all their layers as well as the relationships between them.

Sketch trees can be created in multiple ways given the flexibility and freedom of language. However, some of the elements in the target model have no direct mapping in the source model. One example is the attribution of borders. Layer nodes contain an array of Borders that represent the layer borders. Borders are enabled for every side of the layer. It is not possible to define borders that do not surround all the sides of the layer. Other types of border customization, that do not surround all the sides of the layer, are not supported by the Sketch syntax. Designers usually introduce new layers resulting in a wide variety of trees for each widget/UI pattern. This technique makes it particularly difficult to identify layers and consequently have a uniform layer processing method. After customization, UI Patterns and Widgets trees may have no resemblance to the original structure defined in the OutSystems Live Style Guide Sketch document or with the web technology structure. Due to the complexity of customizing a UI Pattern, currently, only a subset of UI patterns are being customized. For each 


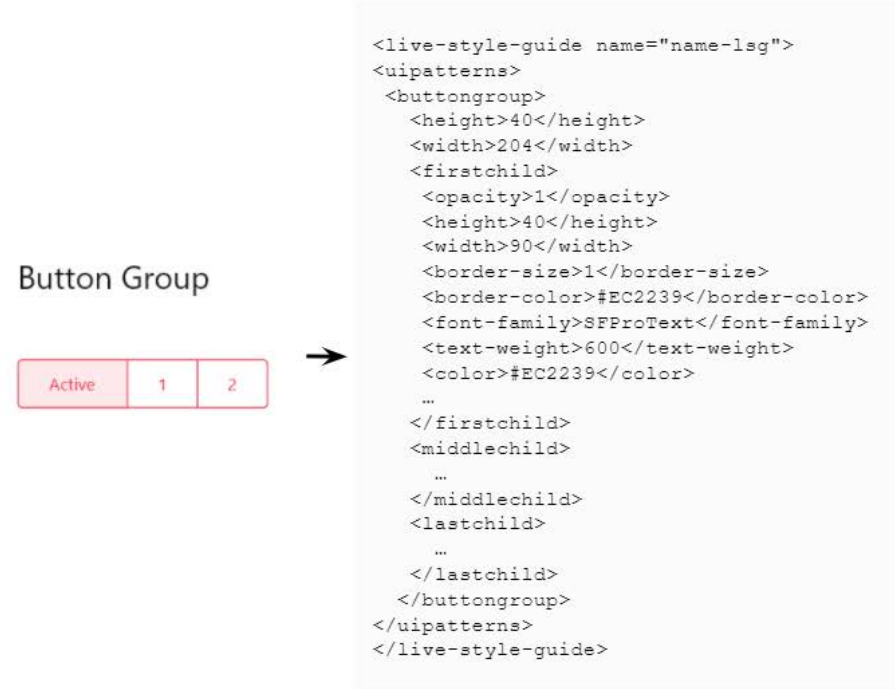

Sketch Representation

Intermediate Representation

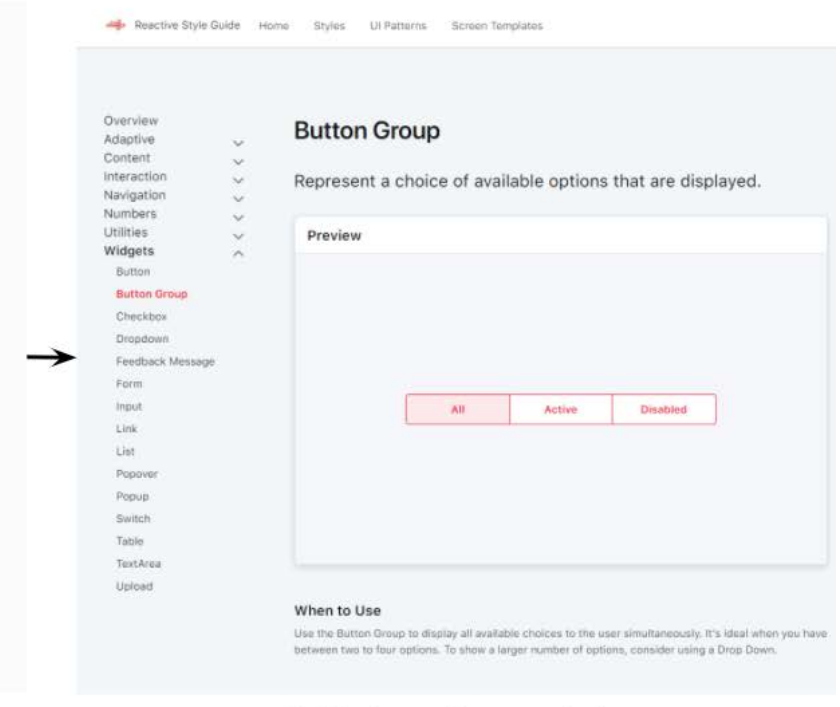

OutSystems Representation

Figure 7: Transformation of the Button Group Component

\begin{tabular}{|l|l|l|}
\hline Active & 1 & 2 \\
\hline
\end{tabular}

Figure 8: OutSystems UI Button Group Widget

UI Pattern, our tool has a tree collection of structures that can be processed, including the default tree for each widget defined in the LSG template.

To map elements represented by a group of layers, we use a granular approach based on the identification of micro-patterns. Instead of handling the element as a whole, we try to find matching atomic reusable elements within its components. This approach eases the tool evolution and improves the return of investment in doing so. If we processed the patterns as a whole and since the processing of all patterns is unreachable, there would be a large number of patterns that would not be customized. This way, we increase the effectiveness of the tool, since even though many patterns are not fully recognized, there is a large number that will be partially recognized and converted.

Frequently, trees have Symbol Instance layers. Symbol Instances are references for Symbol layers defined in the Symbols page. The processing of such layers is done using a lazy approach. Every time a symbol is needed, it is processed and saved in memory for future use. In addition to Symbol Instance layers, trees have other node types namely shape, text, image and group layers. Each type of layer is processed differently, since the relevant elements of each layer vary as illustrated in Layer Attributes.

When a tree or a subtree is not recognized, components are treated as Custom Patterns, that is, a pattern whose structure and
Table 1: Layer Attributes

\begin{tabular}{cc}
\hline Layer Type & \multicolumn{1}{c}{ Attributes } \\
\hline Symbol Instance & $\begin{array}{l}\text { Override Values, Frame } \\
\text { (Height and Weight, X, Y), Shadow } \\
\text { Text }\end{array}$ \\
Frame(X and Y), Line Height, Color, \\
Font, Weight, Size, Shadow, Shared Style \\
Fills (File name), Frame (height \\
Shape & and weight, X, Y), Background \\
& Frame (Height and Weight, X, Y), \\
& Shadow, Border, Background \\
\hline
\end{tabular}

nodes our tool is not able to identify and process and no intermediate representation is generated.

Currently, our tool is capable of transforming a subset of Widgets / UI Patterns identified by the OutSystems front-end experts team as being the most relevant and frequently used and customized. For each one, after identifying and processing micro-patterns, we have to establish the relation between them and create a new processed structure representing it. This work is done individually for each one since we need to know the base structure of the element.

3.7.2 Intermediate Representation Generation. The generated intermediate representation is a XML document inspired by the structure of the XML-based languages analyzed in section 2 . The file is divided into 2 sections: Styles and UI Patterns. The Styles element has the style rules corresponding to the first level of customization. Variables used across the document and elements, such as colors, font sizes and borders, are declared in this section. In the UI Patterns section, all the widgets and UI Patterns are represented. The rules for mapping elements are described in Table 2. 
Table 2: Customization Transformation Framework

\begin{tabular}{|c|c|c|c|}
\hline Rule $N^{o}$ & Source Model & Intermediate & $\begin{array}{l}\text { Web } \\
\text { Technology }\end{array}$ \\
\hline \multicolumn{4}{|l|}{ Layers } \\
\hline 1 & Page & Group & - \\
\hline 2 & Group & $\begin{array}{l}\text { Group } \\
\text { Element }\end{array}$ & - \\
\hline 3 & Text & $\begin{array}{l}\text { Text } \\
\text { Element }\end{array}$ & $\begin{array}{l}\text { CSS } \\
\text { Attributes }\end{array}$ \\
\hline 4 & Shape & Element & $\begin{array}{l}\text { CSS } \\
\text { Attributes }\end{array}$ \\
\hline 5 & Image & $\begin{array}{l}\text { Image } \\
\text { Element }\end{array}$ & $\begin{array}{l}\text { CSS } \\
\text { Attributes }\end{array}$ \\
\hline 6 & $\begin{array}{l}\text { Symbol } \\
\text { Instance }\end{array}$ & Element & $\begin{array}{l}\text { CSS } \\
\text { Declaration }\end{array}$ \\
\hline \multicolumn{4}{|l|}{ Elements } \\
\hline 7 & Point & $\begin{array}{l}\text { Coordinates } \\
+ \text { Radius }\end{array}$ & $\begin{array}{l}\text { Border- } \\
\text { Radius }\end{array}$ \\
\hline 8 & Fills & $\begin{array}{l}\text { Hex Color + } \\
\text { Opacity }\end{array}$ & $\begin{array}{l}\text { Background- } \\
\text { Color }\end{array}$ \\
\hline 9 & Border & $\begin{array}{l}\text { Hex Color }+ \\
\text { Size }\end{array}$ & $\begin{array}{l}\text { Border-size, } \\
\text { border-color }\end{array}$ \\
\hline 10 & Shadow & $\begin{array}{l}\text { Spread, Offset x } \\
\text { Offset y, Color }\end{array}$ & $\begin{array}{l}\text { Box- } \\
\text { Shadow }\end{array}$ \\
\hline 11 & Blur & $\begin{array}{l}\text { radius, blur } \\
\text { type, saturation }\end{array}$ & Filter \\
\hline 12 & Text Font & Font Name & $\begin{array}{l}\text { Font-Family } \\
\text { Font-Weight }\end{array}$ \\
\hline 13 & $\begin{array}{l}\text { Paragraph } \\
\text { Style }\end{array}$ & Text Element & $\begin{array}{l}\text { Alignment } \\
\text { Line-Height }\end{array}$ \\
\hline 14 & Text Color & Hex Color & Color \\
\hline
\end{tabular}

Layer Pages are represented in the intermediate representation as Groups. Group and Symbol Instance Layers are Group Elements.

The Text Elements described in Rule $\mathrm{n}^{\circ} 3$ are the representation of the source model's Text Layers. These elements are characterized by a group of attributes, namely font family, weight, size, line height and coordinates $\mathrm{X}$ and $\mathrm{Y}$.

UI Patterns are represented in our Intermediate Representation as compositions of Text Elements and Layer Elements.

3.7.3 Customization Delta Calculation. After the generation of the Intermediate Representation, we need to identify the difference between the Sketch instance and the LSG template. We call the difference, the Customization $\Delta$.
Since the LSG Sketch template represents the OutSystems UI default theme, the changes made on top of this document represent the customization done. This calculation will separate the customization from the OutSystems UI base.

The $\Delta$ is identified by comparing the intermediate representation of the OutSystems LSG Sketch file with the intermediate representation of the customized instance. The difference between the two documents is used to customize the applications.

\subsection{Customization Delta to Target Model}

As explained in section 3.5, OutSystems applications inherit the default OutSystems UI CSS styling rules. Customizing applications can be done through the OutSystems IDE interface or by adding new CSS declarations that override the default ones.

Since the elements present in the Sketch file represent the default OutSystems UI components, mapping the $\Delta$ elements to OutSystems implies mapping the only the customizations done to these elements to the corresponding CSS rules. During this stage, we compare the values declared in the OUtSystems UI CSS style sheet for each rule with the corresponding $\Delta$ elements. The relationship between these components and the corresponding OutSystems UI Style sheet CSS Selectors had to be previously established in order to compare the values. CSS Declarations may be hierarchical and one CSS Selector may affect multiple $\Delta$ elements. For this reason, mapping is not straightforward since one CSS selector may correspond to multiple $\Delta$ elements. One example of this is the selector .btn that affects all button instances.

When the customization $\Delta$ does not contain the representation of a certain item, its structure is not recognized. In this case, a note is added to the report file mentioning that the component needs to be verified and customized manually.

In contrast, when a $\Delta$ element exists however its content is nonexistent, no differences were identified between the customized element and the corresponding OutSystems UI base after the $\Delta$ generation explained in subsubsection 3.7.3. In this case, the element is not compared with the corresponding CSS rules since no adjustments were made and the component only inherits the OutSystems UI styling. In the report file, the item is marked as reviewed.

Finally, if the customization $\Delta$ contains an element and a group of attributes that describe it, the corresponding OutSystems component needs to be customized. The attribute values are compared with the CSS rules values. New CSS selector and corresponding declarations are added to the style sheet when the values are different.

A CSS style sheet is the result artifact of this stage. The style sheet which is later added and referenced in the applications modules.

\subsection{Target Model Instance Generation}

The last task performed by our tool is the generation of the target model instance, two customized LSG OutSystems applications. Outsystems applications are generated with the help of an OutSystems API that allows the manipulation of its model. The applications are created from a starter base application representing the default Live Style Guide and Theme applications. Applications and their modules are personalized with the customer name and the corresponding logo provided by the user. The CSS file generated 


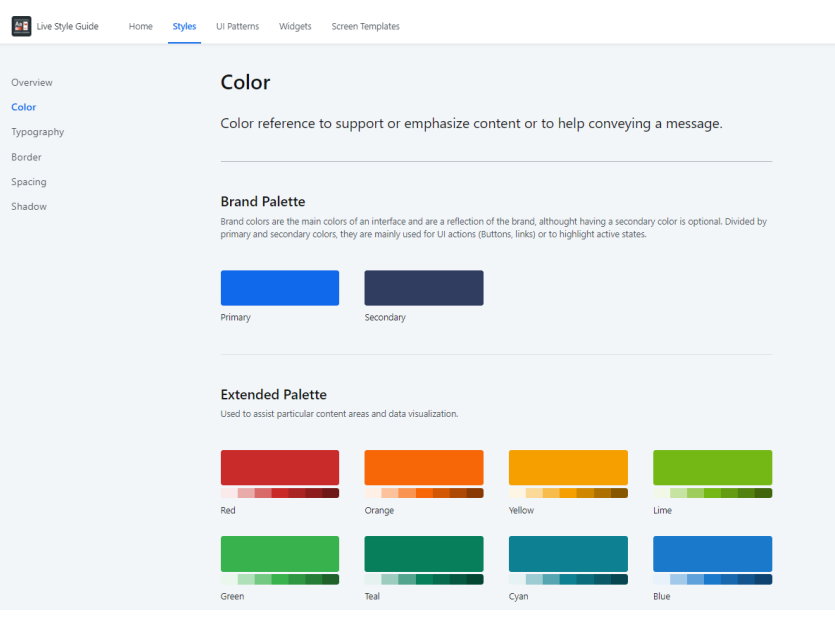

Figure 9: Sample OutSystems Live Style Guide

in the previous step is added to the Theme module of the Theme application overriding its default look-and-feel.

For the generated project to be correct, it is necessary that fonts non existing by default in the OutSystems IDE and declared in the Sketch file be supplied by the user. Those files are added as resources to the theme module of the Theme application. An example of a customized OutSystems LSG application is shown in Figure 9.

The OutSystems language has extensibility points that can be used to enrich applications. We can take advantage of those points to inject custom CSS into applications and complement the generated code.

If there are manual changes to the generated OutSystems code (e.g. for further tweaks or to accommodate a complex custom pattern) and there is a need to re-generate the code because of changes to the Sketch design, people need to be careful, otherwise they may lose the manual changes because of the newly generated code. The loss of manual customizations can be easily avoided if people leverage the merge capabilities of the OutSystems platform - these allow the user to cherry pick the relevant changes from both versions (the one with manual changes and the one resulting from the design file changes).

\section{EVALUATION}

To evaluate the results of our approach, we made available a web application to the OutSystems Front-End Experts team. This team is composed of 11 professional developers, whose daily work is dedicated to dealing with complex front-end topics. Part of their responsibility is materializing the UX/UI designs of enterprise-grade applications into hand-crafted Style Guides and samples.

While developing the tool, we leveraged the artifacts (designs as well as resulting style guides and sample pages) of 10 recently completed enterprise projects. This made it possible to validate the correct behaviour of the tool in real-life scenarios. Furthermore, these past projects were great references against which to evaluate not only the tool effectiveness and impact but also the quality of the resulting code (e.g. compliance with established programming practices). To evaluate the actual impact of our tool, we asked the
Front End Experts team to evaluate and compare the results of their own work in these past projects against the results obtained by using our tool. While the results are still qualitative in nature, we believe the informed opinion of a group of domain experts is invaluable, especially considering the complex and non-linear nature of the process through which UX/UI designs get transformed into style guides.

Based on feedback collected so far, preliminary conclusions point that for projects without custom patterns, the tool can reduce the time the Front End team needs to invest bootstrapping an application's customization by 3 out of 4 days. For projects with higher customization requirements, the conservative expectation is for savings of at least 1 out of 5 days.

Notwithstanding the qualitative nature of the evaluation process, we believe that the results collected so far - $20-75 \%$ savings in time invested by highly specialized and experienced professionals - are very strong indicators that the approach followed with this work is a good fit for dealing with the problems we proposed to tackle initially.

While comparing the manually vs automatically generated artifacts, we concluded that our tool detects small variations of color which were actually not detected during the manual process. This was one of the "small" but annoyingly present problems pointed out by the experts while researching the friction points of the manual process.

It is part of our plans to extend the evaluation of our tool to two companies that specialize in design and front-end activities. This should provide us not only with higher confidence of the tool's impact and effectiveness but also with further feedback on topics to explore in our future work.

\section{CONCLUSIONS AND FUTURE WORK}

Our work is motivated by the currently existing gap in efficiency and effectiveness when it comes to UX and UI designers collaborating with front-end developers. This is a relevant problem today because good UX/UI is an essential market differentiator for most companies, while hiring skilled UX, UI and front-end professionals has been a forever challenging topic.

To mitigate the above challenge, with this article, we presented an approach to automate the conversion of high-fidelity design artifacts into low-code web-technology reusable UI components and applications. The conversion process is based on a unidirectional model transformation and an intermediate representation independent from both technologies.

According to the results obtained, the usage of the developed tool represents a great improvement in the Live Style Guide creation process. Depending on the degree of UX and UI customization, professional teams can save between $20-75 \%$ of the time invested in creating Live Style Guides. If applied at scale to the OutSystems ecosystem, at the end of a year, these savings can translate into up to 12000 days - assuming a customer base exceeding 1200 companies that develop 4 projects per year, lasting 3 months each. The effort saved can be invested in other tasks that represent greater value to the customer. The results obtained highlight the great impact of our tool and the appliance of the used techniques to bridge design and web technologies. 
The biggest challenge so far has been identifying the source model elements since they are context-dependent. Mapping of design to web concepts is not a straightforward process and consequently, each element needs to be identified and processed independently. To overcome the problem, our solution relies on an instance of a design technology meta-model with a well-defined structure as our source model and a dataset of customized components to identify and process components.

In terms of future work, an interesting opportunity to explore is the conversion of whole application pages from their design representation to a web technology. A particular challenge here is safekeeping the referential identity of the components being reused in such compositions.

Last but not least, the current implementation relies on the OutSystems UI framework concepts. Future research could examine the viability of removing this dependency.

\section{ACKNOWLEDGMENTS}

This work has been funded by OutSystems Research and Development and been carried out as part of the collaboration between OutSystems and NOVA LINCS (UID/CEC/04516/2013).

The authors would like to thank the OutSystems Advanced Development team and partner companies who provided insights about their practices and helped evaluate the tool.

\section{REFERENCES}

[1] Marc Abrams, Constantinos Phanouriou Ł, Alan L Batongbacal, Stephen M Williams, and Jonathan E Shuster. 1999. UIML: an appliance-independent XML user interface language. Technical Report.

[2] Quick Base. 2020. Quick Base. Retrieved February 19, 2020 from https://www. quickbase.com/

[3] Tony Beltramelli. 2018. pix2code: Generating code from a graphical user interface screenshot. Proceedings of the ACM SIGCHI Symposium on Engineering Interactive Computing Systems, EICS 2018 (2018), 1-9. https://doi.org/10.1145/3220134. 3220135 arXiv: 1705.07962

[4] Marco Brambilla and Piero Fraternali. 2015. Implementation of applications specified with IFML. Interaction Flow Modeling Language February (2015), 279334. https://doi.org/10.1016/b978-0-12-800108-0.00010-2

[5] Sketch B.V. 2020. Sketch. Retrieved January 20, 2020 from https://www.sketch. com/

[6] Morgan Dixon and James Fogarty. 2010. Prefab: implementing advanced behaviors using pixel-based reverse engineering of interface structure. In Proceedings of the SIGCHI Conference on Human Factors in Computing Systems. 1525-1534.

[7] Figma. 2020. Figma. Retrieved January 20, 2020 from https://www.figma.com/

[8] Saad Hassan, Manan Arya, Ujjwal Bhardwaj, and Silica Kole. 2018. Extraction and Classification of User Interface Components from an Image. Int. F. Pure Appl. Math 118, 24 (2018).

[9] Google Inc. 2020. App Maker - Google Developers. Retrieved February 19, 2020 from https://developers.google.com/appmaker

[10] InVisionApp Inc. 2020. InVision / Digital product design, workflow collaboration Retrieved January 20, 2020 from https://www.invisionapp.com/

[11] Kissflow Inc. 2020. Kissflow - Digital Workplace. Retrieved February 19, 2020 from https://kissflow.com/

[12] Zecoda Inc. 2020. Zecoda Turn Designs Into Code Automatically. Retrieved January 12, 2020 from https://zecoda.com/

[13] Hi Interactive. 2020. Hi Interactive / User-centred experiences. Retrieved February 18, 2020 from https://www.hi-interactive.com/

[14] Vanita Jain, Piyush Agrawal, Subham Banga, Rishabh Kapoor, and Shashwat Gulyani. 2019. Sketch2Code: Transformation of Sketches to UI in Real-time Using Deep Neural Network. (2019), 1-15. arXiv:1910.08930 http://arxiv.org/abs/1910. 08930

[15] Jang-geun Ki and Kee-young Kwon. 2019. Detection of GUI Elements on Sketch Images Using Object Detector Based on Deep Neural Networks. 502, January (2019), 10-13. https://doi.org/10.1007/978-981-13-0311-1

[16] Q. Limbourg, Jean Vanderdonckt, B. Michotte, L. Bouillon, and Víctor LópezJaquero. 2005. USIXML: A Language Supporting Multi-path Development of User Interfaces Engineering Human Computer Interaction and Interactive Systems. Lecture Notes in Computer Science 3425 (01 2005), 134-135.
[17] Neonto Ltd. 2020. React Studio. Retrieved January 13, 2020 from https: //reactstudio.com/

[18] Mediaweb. 2020. Mediaweb / Agile the Modern Web. Retrieved February, 18, 2020 from https://mediaweb.pt/

[19] Tuan Anh Nguyen and Christoph Csallner. 2016. Reverse engineering mobile application user interfaces with REMAUI. Proceedings - 2015 30th IEEE/ACM International Conference on Automated Software Engineering, ASE 2015 (2016), 248-259. https://doi.org/10.1109/ASE.2015.32

[20] Outsystems. 2020. OutSystems. Retrieved July 10, 2020 from https://outsystems. $\mathrm{com} /$

[21] PaintCode. 2020. PaintCode - Turn your drawings into Objective-C or Swift drawing code. Retrieved January 12, 2020 from https://www.paintcodeapp.com/

[22] Angel Puerta and Jacob Eisenstein. 2001. XIML: A Universal Language for User Interfaces User interface languages, model-based systems, user-interface management systems, interface models INTRODUCTION. Technical Report.

[23] Nathalie Souchon and Jean Vanderdonckt. 2003. A review of XML-compliant user interface description languages. Lecture Notes in Computer Science (including subseries Lecture Notes in Artificial Intelligence and Lecture Notes in Bioinformatics) 2844, May (2003), 377-391. https://doi.org/10.1007/978-3-540-39929-2_26

[24] Supernova Studio. 2020. Supernova Studio / The World's First Design to Code Platform. Retrieved January 13, 2020 from https://supernova.io/

[25] Sarah Suleri, Vinoth Pandian Sermuga Pandian, Svetlana Shishkovets, and Matthias Jarke. 2019. Eve: A sketch-based software prototyping workbench. In Extended Abstracts of the 2019 CHI Conference on Human Factors in Computing Systems. 1-6.

[26] Anima ToolKit. 2020. Anima ToolKit - Sketch to HTML. Retrieved January 12, 2020 from https://www.animaapp.com/

[27] TrackVia. 2020. TrackVia/The Most-Trusted Application Building Platform. Retrieved February 19, 2020 from https://trackvia.com/

[28] Nintex Workflow. 2020. Workflow Automation Software Simplified - Nintex Workflow. Retrieved February 19, 2020 from https://www.nintex.com/workflowautomation/advanced-workflow/

[29] Yotako. 2020. Yotako. Retrieved January 13, 2020 from https://www.yotako.io/

[30] Zoho. 2020. Online app maker / Create custom apps for your business - Zoho Creator. Retrieved February 19, 2020 from https://www.zoho.com/creator/ 